\title{
Van der Waals-like Equations of State. II. Application to $n$-Alkane Mixtures and Poly(dimethylsiloxane) Solutions
}

\author{
Koichi Fujisawa, Tomoo Shiomi, Fumiyuki Hamada, ${ }^{*}$ \\ Akio NAKAJIMA, and Jang-oo LEE \\ Department of Polymer Chemistry, Faculty of Engineering, \\ Kyoto University, Kyoto 606, Japan.
}

(Received January 16, 1981)

\begin{abstract}
The FHN equation of state proposed in a previous paper has also been applied to mixtures of $n$-alkanes. The values of excess enthalpy, volume, and chemical potential obtained from the FHN equation using the reduction parameters independent of temperature were somewhat different from those derived from the Flory theory which uses reduction parameters dependent on temperature. The interaction parameters $\chi$ and excess volumes $V^{E}$ calculated from six equations of state including the FHN equation, were compared with each another for solutions of poly(dimethylsiloxane) in various solvents, under the assumption that the combining rule for mixing given by Flory is valid. No equations gave agreement between the observed and calculated values of $\chi$ and $V^{E}$.

KEY WORDS Interaction Parameter / Excess Volume / $n$-Alkane Mixtures / Poly(dimethylsiloxane) Solutions /
\end{abstract}

In our preceding paper, ${ }^{1}$ we tested six representative equations of state, the van der Waals, Flory, ${ }^{2-4}$ FHN, ${ }^{1}$ Guggenheim, ${ }^{5}$ Frisch, ${ }^{6}$ and Thiele $^{7}$ equations, going on the assumption of the validity of the corresponding states principle.

It was shown that the FHN equation was more suitable for various pure liquids as well as $n$-alkane liquids than the other five equations when combined with the van der Waals type of the attractive energy, and that the Flory equation was also good. The reduction parameters obtained from the FHN equation were found to be somewhat dependent on temperature when evaluated from data for the specific volume $v_{\text {sp }}$, the thermal expansivity $\alpha$, and the thermal pressure co-efficient $\gamma$. Encouraged by this interesting result, in this paper, we examine how well the FHN equation can reproduce the experimental excess thermodynamic quantities for mixtures of $n$-alkanes when the reduction parameters independent of temperature are used.

We also examine the applicability of the six

\footnotetext{
* To whom correspondence should be addressed.
}

equations to poly(dimethylsiloxane) (PDMS) solutions. It has been reported ${ }^{8-12}$ that the Flory theory ${ }^{2-4}$ does not give agreement with the observed values of the interaction parameter $\chi$ and the excess volume $V^{E}$ for solutions of this polymer. In this paper, as a step to clarify sources of this discrepancy, $\chi$ and $V^{E}$ calculated from the six equations are compared with experimental values for PDMS systems, under the assumption that the combining rule for mixing given by Flory $^{2-4}$ is valid.

\section{THEORETICAL}

According to the reduced form given by Flory, ${ }^{2-4}$ the generalized partition function for the system comprising $N r$-mers can be written,

$$
Z=Z_{\text {comb }}\left(g v^{*}\right)^{r N c} Q(\tilde{v})^{r N c} \exp (r N c / \tilde{v} \tilde{T})
$$

where $Z_{\text {comb }}$ is the combinatorial entropy part, $g$ is a geometric factor, $v^{*}$ is the characteristic volume, $\tilde{v}$ and $\tilde{T}$ are the reduced volume and temperature, respectively, $c$ is the number of external degrees of 
freedom, and $Q(\tilde{v})$ is related to $H(\tilde{v})$ given by eq I$14^{* 1}$ as follows:

$$
H(\tilde{v}) / \tilde{v}=[\partial \ln Q(\tilde{v}) / \partial \tilde{v}]_{\tilde{T}}
$$

For a binary mixture, we use the following assumptions given by Flory ${ }^{2-4}$ : (i) mixing is random, (ii) the external degree of freedom for the mixture is given by a linear combination with that for the pure components, and (iii) $v_{1}{ }^{*}=v_{2}{ }^{*}=v^{*}$. The characteristic pressure $p^{*}$ and temperature $T^{*}$ for the mixture are thus given by,

$$
p^{*}=\varphi_{1} p_{1}^{*}+\varphi_{2} p_{2}{ }^{*}-\varphi_{1} \theta_{2} X_{12}
$$

and

$$
1 / T^{*}=\left(\varphi_{1} p_{1}{ }^{*} / T_{1}{ }^{*}+\varphi_{2} p_{2}{ }^{*} / T_{2}{ }^{*}\right) / p^{*},
$$

where $X_{12}$ is the exchange enthalpy parameter, $\varphi_{i}$ is the segment fraction of component $i(i=1,2)$ defined by,

$$
\varphi_{i}=r_{i} N_{i} /\left(r_{1} N_{1}+r_{2} N_{2}\right)
$$

and $\theta_{2}$ is the site fraction given by,

$$
\theta_{2}=\frac{\left(s_{2} / s_{1}\right) \varphi_{2}}{\varphi_{1}+\left(s_{2} / s_{1}\right) \varphi_{2}}
$$

where $s_{2} / s_{1}$ is the ratio of the number of contact sites per segment.

Assuming that eq 1 for $Z$ is applicable to the mixture, and using the Flory-Huggins expression of the combinatorial entropy, we can obtain the chemical potential of component 1 at $p=0$ as,

$$
\begin{aligned}
\left(\mu_{1}-\mu_{1}{ }^{\circ}\right)= & R T\left[\ln \left(1-\varphi_{2}\right)+\left(1-r_{1} / r_{2}\right) \varphi_{2}\right] \\
& +p^{*} V_{1} *\left\{\tilde{T}_{1} \ln \left[Q\left(\tilde{v}_{1}\right) / Q(\tilde{v})\right]\right. \\
& \left.+\left(\tilde{v}_{1}^{-1}-\tilde{v}^{-1}\right)\right\}+\left(V_{1}{ }^{*} X_{12} / \tilde{v}\right) \theta_{2}{ }^{2}
\end{aligned}
$$

where $V_{1}{ }^{*}$ is the molar characteristic volume of component 1 . The excess chemical potential is defined by,

$$
\left(\mu_{1}-\mu_{1}^{\circ}\right)^{E}=\left(\mu_{1}-\mu_{1}^{\circ}\right)-R T \ln x_{1}
$$

where $x_{1}$ is the mole fraction of component 1 . Further, the interaction parameter $\chi$ familiar in the theory of polymer solutions can be written,

$$
\begin{aligned}
\chi= & {\left[\left(\mu_{1}-\mu_{1}^{\circ}\right) / R T-\ln \left(1-\varphi_{2}\right)-\left(1-r_{1} / r_{2}\right) \varphi_{2}\right] / \varphi_{2}{ }^{2} } \\
= & \left(p_{1}{ }^{*} V_{1}{ }^{*} / R T \varphi_{2}{ }^{2}\right)\left\{\tilde{T} \ln \left[Q\left(\tilde{v}_{1}\right) / Q(\tilde{v})\right]\right. \\
& \left.\quad+\left(\tilde{v}_{1}^{-1}-\tilde{v}^{-1}\right)\right\}+\left(V_{1}^{*} X_{12} / \tilde{v} R T\right)\left(\theta_{2} / \varphi_{2}\right)^{2}
\end{aligned}
$$

At infinite dilution, we have,

$$
\begin{aligned}
\chi_{1} & =\lim _{\varphi_{2} \rightarrow 0} \chi \\
& =\left(p_{1} * V_{1}^{*} / \tilde{v}_{1} R T\right)\left(A^{2} \alpha_{1} T / 2+Y_{12}\right)
\end{aligned}
$$

where,

$$
\begin{aligned}
A & =\left(1-T_{1}{ }^{*} / T_{2}{ }^{*}\right)\left(p_{2}{ }^{*} / p_{1}{ }^{*}\right)-\left(s_{2} / s_{1}\right)\left(X_{12} / p_{1}{ }^{*}\right) \\
Y_{12} & =\left(s_{2} / s_{1}\right)^{2}\left(X_{12} / p_{1}{ }^{*}\right)
\end{aligned}
$$

The enthalpy change of mixing $\Delta H^{M}$ can be written as,

$$
\begin{aligned}
\Delta H^{M}= & N_{1} p_{1} * V_{1} *\left(1 / \tilde{v}_{1}-1 / \tilde{v}\right) \\
& +N_{2} p_{2}{ }^{*} V_{2} *\left(1 / \tilde{v}_{2}-1 / \tilde{v}\right)+\left(N_{1} V_{1} * \theta_{2} / \tilde{v}\right) X_{12}
\end{aligned}
$$

and the enthalpy of mixing $\Delta H^{M}(\infty)$ at infinite dilution of component 2 is,

$$
\begin{aligned}
\Delta H^{M}(\infty)= & \left(v_{\mathrm{sp}, 2}^{*} / \tilde{v}_{1}\right)\left[X_{12}\left(s_{2} / s_{1}\right)\right. \\
& \left.+p_{2}{ }^{*}\left(\tilde{v}_{1} / \tilde{v}_{2}-1\right)-\alpha_{1} T p_{1}{ }^{*} A\right]
\end{aligned}
$$

where $v_{\mathrm{sp}, 2}^{*}$ is the specific characteristic volume of component 2 .

The excess volume $V^{E}$ is given by,

$$
V^{E} / V^{0}=\tilde{v} /\left(\varphi_{1} \tilde{v}_{1}+\varphi_{2} \tilde{v}_{2}\right)-1
$$

where $V^{0}$ is the sum of volumes of both components.

\section{COMPARISON OF THEORIES WITH EXPERIMENTS}

\section{n-Alkane Mixtures}

We need to evaluate the reduction parameters for pure liquids in order to obtain the thermodynamic quantities of mixtures. Following Flory, ${ }^{2-4}$ we can evaluate the reduction parameters from the specific volume $v_{\mathrm{sp}}$, the thermal expansivities $\alpha$ and the thermal pressure coefficient $\gamma$. If the FHN equation is used, $T^{*}$ and $v_{\mathrm{sp}}^{*}$ for $n$-alkanes are given by, ${ }^{1}$

$$
T^{*}=6098\left[1-4.042(1 / r)+7.73(1 / r)^{2}\right]-(15.36 / r) t
$$

and
*1 Hereafter, equations taken from the preceding paper will be preceded by the numeral I. 
Van der Waals-like Equation of State

Table I. Observed and calculated excess volumes of equimolar mixtures

\begin{tabular}{|c|c|c|c|c|c|c|c|}
\hline \multirow{2}{*}{ Mixture } & \multirow{2}{*}{$\frac{\text { Temp }}{{ }^{\circ} \mathrm{C}}$} & \multicolumn{2}{|c|}{$V^{\mathrm{E}} / \mathrm{cm}^{3} \mathrm{~mol}^{-1}$} & \multirow{2}{*}{ Mixture } & \multirow{2}{*}{$\frac{\text { Temp }}{{ }^{\circ} \mathrm{C}}$} & \multicolumn{2}{|c|}{$V^{\mathrm{E}} / \mathrm{cm}^{3} \mathrm{~mol}^{-1}$} \\
\hline & & Obsd $^{a}$ & Calcd & & & Obsd $^{a}$ & Calcd \\
\hline \multirow{3}{*}{$\mathrm{C}_{6}+\mathrm{C}_{12}$} & 20 & -0.31 & -0.44 & \multirow{5}{*}{$\mathrm{C}_{8}+\mathrm{C}_{16}$} & 30 & -0.21 & -0.29 \\
\hline & 25 & -0.35 & -0.47 & & 50 & -0.33 & -0.38 \\
\hline & 35 & -0.38 & -0.51 & & 51 & -0.32 & -0.38 \\
\hline \multirow{10}{*}{$\mathrm{C}_{6}+\mathrm{C}_{16}$} & \multirow[t]{2}{*}{20} & -0.49 & \multirow{2}{*}{-0.65} & & 76 & -0.48 & -0.52 \\
\hline & & -0.48 & & & 106 & -0.74 & -0.76 \\
\hline & \multirow[t]{3}{*}{25} & -0.58 & \multirow{2}{*}{-0.70} & $\mathrm{C}_{8}+\mathrm{C}_{24}$ & 106 & -1.33 & -1.31 \\
\hline & & -0.53 & & $\mathrm{C}_{8}+\mathrm{C}_{32}$ & 96 & -1.49 & -1.45 \\
\hline & & -0.58 & -0.75 & & 106 & -1.70 & -1.63 \\
\hline & 35 & -0.62 & -0.81 & $\mathrm{C}_{8}+\mathrm{C}_{36}$ & 96 & -1.62 & -1.55 \\
\hline & 40 & -0.69 & -0.86 & & 106 & -1.85 & -1.75 \\
\hline & 50 & -0.82 & -0.99 & $\mathrm{C}_{9}+\mathrm{C}_{16}$ & 126 & -0.61 & -0.59 \\
\hline & 51 & -0.82 & -1.00 & $\mathrm{C}_{9}+\mathrm{C}_{24}$ & 51 & -0.43 & -0.48 \\
\hline & 60 & -0.97 & -1.14 & & 76 & -0.63 & -0.65 \\
\hline $\mathrm{C}_{6}+\mathrm{C}_{24}$ & 51 & -1.19 & -1.41 & & 96 & -0.84 & -0.82 \\
\hline \multirow[t]{2}{*}{$\mathrm{C}_{7}+\mathrm{C}_{12}$} & 25 & -0.17 & -0.24 & & 106 & -0.96 & -0.92 \\
\hline & 35 & -0.20 & -0.29 & & 126 & -1.28 & -1.17 \\
\hline \multirow[t]{4}{*}{$\mathrm{C}_{7}+\mathrm{C}_{16}$} & 20 & -0.31 & -0.41 & $\mathrm{C}_{9}+\mathrm{C}_{32}$ & 96 & -1.14 & -1.07 \\
\hline & 25 & -0.34 & -0.44 & $\mathrm{C}_{9}+\mathrm{C}_{36}$ & 96 & -1.25 & -1.17 \\
\hline & 40 & -0.45 & -0.53 & & 106 & -1.41 & -1.31 \\
\hline & 76 & -0.77 & -0.86 & & 126 & -1.89 & -1.65 \\
\hline $\mathrm{C}_{7}+\mathrm{C}_{24}$ & 76 & -1.22 & -1.33 & $\mathrm{C}_{9}+\mathrm{C}_{62}$ & 126 & -2.51 & -2.10 \\
\hline $\mathrm{C}_{7}+\mathrm{C}_{36}$ & 76 & -1.56 & -1.67 & $\mathrm{C}_{10}+\mathrm{C}_{16}$ & 20 & -0.07 & -0.09 \\
\hline $\mathrm{C}_{8}+\mathrm{C}_{16}$ & 20 & -0.19 & -0.25 & & 30 & -0.09 & -0.11 \\
\hline
\end{tabular}

a Reference 13, in which the works of some investigators are summarized by Orwoll and Flory.

$$
\begin{aligned}
v_{\mathrm{sp}}^{*}= & 0.8737+0.6895(1 / r) \\
& -\left(1.12 \times 10^{-2} / r\right)\left[4.74 \times 10^{-2}+(1 / r)\right] t,
\end{aligned}
$$

respectively, where $r$ is the number of segments per molecule, $r=n+1$ (where $n$ is the number of carbon atoms), and $t$ is the temperature. The external degree of freedom per gram, $c_{\mathrm{sp}}$, is given by,

$$
c_{\mathrm{sp}}=0.01061+0.05808 /(r-1)
$$

The reduction parameter $p^{*}$ can be calculated by $p^{*}=c_{\mathrm{sp}} T^{*} R / v_{\mathrm{sp}}^{*}$.

As was shown in the preceding paper, ${ }^{1}$ the temperature dependence of the reduction parameters obtained from the FHN equation is not as much as that obtained from other equations of state. Therefore, we here use the values calculated at $100^{\circ} \mathrm{C}$ from eq $16-18$. The reduced volume $\tilde{v}$ and temperature $\tilde{T}$ can be obtained from eq I-21 and I27.

The exchange enthalpy parameter for the binary mixtures of $n$-alkanes is given by, ${ }^{10}$

$$
X_{12}=\Gamma p_{\infty}{ }^{*}\left(1 / r_{1}-1 / r_{2}\right)^{2} /\left(1+s_{\mathrm{e}} / s_{\mathrm{m}} r_{1}\right)\left(1+s_{\mathrm{e}} / s_{\mathrm{m}} r_{2}\right)^{2}
$$

where $p_{\infty}{ }^{*}$ is the pressure reduction parameter extrapolated to $r=\infty, s_{\mathrm{e}}$ and $s_{\mathrm{m}}$ are the number of interation sites per segment for the middle and terminal segments, respectively, and $\Gamma$ is an adjustable parameter. We now take the value of $\Gamma$ as 0.8 and determine $p_{\infty}{ }^{*}$ and $s_{\mathrm{e}} / s_{\mathrm{m}}$ following Orwoll and Flory. ${ }^{13}$

In Table I, the excess volumes calculated from eq 15 by the use of the parameters thus determined are compared with those observed. The average deviation of the calculated values from the observed ones is $0.10 \mathrm{~cm}^{3} \mathrm{~mol}^{-1}$. Orwoll and Flory ${ }^{13}$ calculated the excess volumes $V^{E}$ from the Flory equation using the parameters dependent on temperature. The average deviation of their calculated results from the observed ones is $0.09 \mathrm{~cm}^{3} \mathrm{~mol}^{-1}$, which is as large as ours. This shows that the FHN equation can reproduce the experimental values fairly well without using the parameters dependent on temperature. 
Table II. Observed and calculated excess enthalpies

\begin{tabular}{|c|c|c|c|c|c|}
\hline \multirow{2}{*}{ Component 1} & \multirow{2}{*}{ Component 2} & \multirow{2}{*}{$x^{2}$} & \multirow{2}{*}{$\frac{\text { Temp }}{{ }^{\circ} \mathrm{C}}$} & \multicolumn{2}{|c|}{$H^{\mathrm{M}} / \mathrm{cal} \mathrm{mol}^{-1}$} \\
\hline & & & & Obsd $^{a}$ & Calcd \\
\hline $\mathrm{C}_{6}$ & $\mathrm{C}_{10}$ & 0.50 & 20 & 4 & 5 \\
\hline $\mathrm{C}_{6}$ & $\mathrm{C}_{12}$ & 0.50 & 20 & 11 & 5 \\
\hline \multirow{9}{*}{$\mathrm{C}_{6}$} & $\mathrm{C}_{16}$ & 0.50 & 20 & 31,30 & 19 \\
\hline & & 0.50 & 25 & 27 & 18 \\
\hline & & 0.50 & 30 & 23 & 17 \\
\hline & & 0.50 & 40 & 16,15 & 15 \\
\hline & & 0.50 & 50 & 8 & 13 \\
\hline & & 0.50 & 51 & 9 & 12 \\
\hline & & 0.50 & 60 & 4 & 10 \\
\hline & & 0.50 & 76 & -4 & 4 \\
\hline & & 0.50 & 100 & -23 & -6 \\
\hline \multirow[t]{3}{*}{$\mathrm{C}_{6}$} & $\mathrm{C}_{24}$ & 0.50 & 51 & 33 & 27 \\
\hline & & 0.50 & 60 & 22 & 23 \\
\hline & & 0.50 & 76 & 6 & 16 \\
\hline $\mathrm{C}_{6}$ & $\mathrm{C}_{36}$ & 0.50 & 76 & 28 & 30 \\
\hline \multirow[t]{4}{*}{$\mathrm{C}_{7}$} & $\mathrm{C}_{16}$ & 0.50 & 20 & 26,25 & 14 \\
\hline & & 0.50 & 25 & 23 & 14 \\
\hline & & 0.33 & 25 & 21 & 14 \\
\hline & & 0.50 & 50 & 10 & 10 \\
\hline $\mathrm{C}_{7}$ & $\mathrm{C}_{36}$ & 0.50 & 76 & 31 & 29 \\
\hline \multirow[t]{4}{*}{$\mathrm{C}_{8}$} & $\mathrm{C}_{16}$ & 0.50 & 20 & 21 & 10 \\
\hline & & 0.36 & 20 & 18 & 10 \\
\hline & & 0.36 & 30 & 13 & 9 \\
\hline & & 0.36 & 50 & 8 & 7 \\
\hline \multirow[t]{4}{*}{$\mathrm{C}_{8}$} & $\mathrm{C}_{24}$ & 0.50 & 51 & 28 & 19 \\
\hline & & 0.50 & 76 & 10 & 15 \\
\hline & & 0.50 & 96 & -1 & 10 \\
\hline & & 0.50 & 106 & -6 & 7 \\
\hline $\mathrm{C}_{8}$ & $\mathrm{C}_{32}$ & 0.50 & 76 & 23 & 23 \\
\hline $\mathrm{C}_{9}$ & $\mathrm{C}_{36}$ & 0.50 & 76 & 29 & 24 \\
\hline \multirow[t]{3}{*}{$\mathrm{C}_{10}$} & $\mathrm{C}_{16}$ & 0.50 & 20 & 12,10 & 5 \\
\hline & & 0.40 & 30 & 8 & 5 \\
\hline & & 0.40 & 73 & 4 & 4 \\
\hline $\mathrm{C}_{16}$ & $\mathrm{C}_{36}$ & 0.50 & 76 & 14 & 8 \\
\hline
\end{tabular}

a Reference 13, in which the works of some investigators are summarized by Orwoll and Flory.

Table II compares the calculated and observed values for the enthalpy of mixing $\Delta H^{M}$. The average deviation of the calculated values from the observed ones is about $6 \mathrm{cal} \mathrm{mol}^{-1}$, which is the same as that obtained by Orwoll and Flory. ${ }^{13}$

For the excess chemical potential, the FHN equation is incapable of reproducing the experimental values without the adjustable entropy parameter $Q_{12}$, as in the Flory theory, ${ }^{13}$ though the value of $Q_{12}$ is small.

\section{Mixtures of Poly (dimethylsiloxane) and Solvent}

In this section, we compare the interaction parameter $\chi$ and the excess volume $V^{E}$ calculated from the six equations with those observed for PDMS in various solvents. The calculation was carried out using the reduction and reduced parameters obtained from $v_{\mathrm{sp}}, \alpha$, and $\gamma$ at each temperature and the exchange enthalpy parameter $X_{12}$ evaluated from the experimental value of $\Delta H^{M}(\infty)$. The values of $s_{2} / s_{1}$ were estimated from the surface areas of both segments by the molecular models and the characteristic volumes. The values of $X_{12}$ obtained from 
$\Delta H^{M}(\infty)$ were negative for the PDMS-octamethylcyclotetrasiloxane (OMCTS) system, ${ }^{12}$ but they were used in the calculation because the absolute

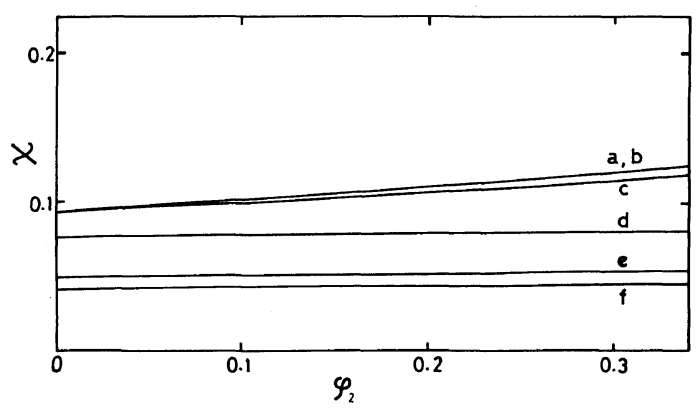

Figure 1. Interaction parameter $\chi v s$. the segment fraction $\varphi_{2}$ for PDMS-octamethylcyclotetrasiloxane at $25^{\circ} \mathrm{C}$ : a, Guggenheim equation; b, Frisch equation; c, Thiele equation; d, FHN equation; e, Flory equation; $f$, van der Waals equation. values of $X_{12}$ cannot be neglected in some equations and because the results obtained by the same method should be compared with each other.

In Figure 1, the concentration dependences of $\chi$ calculated from the six equations for the PDMSOMCTS system at $25^{\circ} \mathrm{C}$ are compared with each other. The value of $\chi$ calculated from the van der Waals equation is the smallest, while the value from the Guggenheim equation is the largest, though the difference between the two is small. The equation which gives a larger value of $\chi$ leads to a larger concentration dependence of $\chi$.

Table III compares the calculated values of $\chi_{1}$ and $V^{E} / V^{0}$ with the experimental values for PDMS in various solvents. For each system, all equations examined give nearly the same values of $\chi_{1}$ which deviate markedly from the experimental values. The calculated values of $V^{E} / V^{0}$ is different in sign from

Table III. Comparison of $V^{E} / V^{0}$ at $\varphi_{2}=0.5$ and $\chi_{1}$ calculated from the six equations with experimental values for PDMS-solvent systems

\begin{tabular}{|c|c|c|c|c|c|c|}
\hline \multirow{2}{*}{ Solvent } & \multicolumn{2}{|c|}{ OMCTS } & \multicolumn{2}{|c|}{ Benzene } & \multicolumn{2}{|c|}{ Cyclohexane } \\
\hline & $\chi_{1}$ & $V^{E} / V^{0} \times 10^{2}$ & $\chi_{1}$ & $V^{E} / V^{0} \times 10^{2}$ & $\chi_{1}$ & $V^{E} / V^{0} \times 10^{2}$ \\
\hline Temp ${ }^{\circ} \mathrm{C}$ & 25 & 25 & 25 & 25 & 25 & 25 \\
\hline Experiment $^{\mathrm{a}}$ & $0.355^{\mathrm{b}}$ & $-0.039^{b}$ & $0.481^{\mathrm{c}}$ & $-0.061^{\mathrm{c}}$ & $0.429^{c}$ & $0.53^{\mathrm{c}}$ \\
\hline $\mathrm{vdW}$ & 0.0414 & 0.002 & 0.223 & 0.895 & 0.124 & 0.500 \\
\hline Flory & 0.0499 & 0.016 & 0.223 & 0.976 & 0.126 & 0.573 \\
\hline FHN & 0.0765 & 0.095 & 0.220 & 1.39 & 0.130 & 0.878 \\
\hline Guggenheim & 0.0938 & 0.315 & 0.208 & 2.17 & 0.128 & 1.51 \\
\hline Frisch & 0.0933 & 0.332 & 0.207 & 2.23 & 0.127 & 1.55 \\
\hline Thiele & 0.0921 & 0.292 & 0.208 & 2.13 & 0.128 & 1.46 \\
\hline
\end{tabular}

\begin{tabular}{|c|c|c|c|c|c|c|}
\hline \multirow{2}{*}{ Solvent } & \multicolumn{2}{|c|}{ Chlorobenzene } & \multicolumn{2}{|c|}{ MEK } & \multicolumn{2}{|c|}{$n$-Hexane } \\
\hline & $\chi_{1}$ & $V^{E} / V^{0} \times 10^{2}$ & $\chi_{1}$ & $V^{E} / V^{0} \times 10^{2}$ & $\chi_{1}$ & $V^{E} / V^{0} \times 10^{2}$ \\
\hline Temp ${ }^{\circ} \mathrm{C}$ & 20 & 25 & 20 & 35 & 20 & 25 \\
\hline Experiment $^{a}$ & $0.477^{\mathrm{d}}$ & $-0.49^{c}$ & $0.500^{\mathrm{e}}$ & $-0.03^{\mathrm{e}}$ & $0.411^{\mathrm{f}}$ & $\begin{array}{l}-0.051^{\mathrm{g}} \\
\left(\varphi_{2}=0.48\right)\end{array}$ \\
\hline vdW & 0.152 & 0.279 & 0.205 & 1.00 & 0.0875 & 0.143 \\
\hline Flory & 0.152 & 0.296 & 0.205 & 1.13 & 0.0966 & 0.205 \\
\hline FHN & 0.151 & 0.368 & 0.206 & 1.60 & 0.123 & 0.492 \\
\hline Guggenheim & 0.150 & 0.492 & 0.197 & 2.58 & 0.140 & 1.22 \\
\hline Frisch & 0.150 & 0.497 & 0.196 & 2.67 & 0.139 & 1.30 \\
\hline Thiele & 0.150 & 0.474 & 0.197 & 2.58 & 0.138 & 1.21 \\
\hline
\end{tabular}

${ }^{a}$ Values of $V^{E} / V^{0}$ except that for $n$-hexane were obtained by interpolation to $\varphi_{2}=0.5 .{ }^{\mathrm{b}}$ Reference $12 .{ }^{\mathrm{c}}$ Reference 8 .

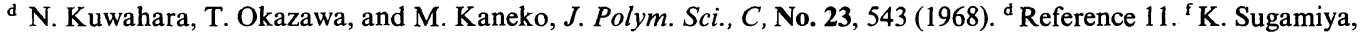
N. Kuwahara, and M. Kaneko, Macromolecules, 7, 66 (1974). ${ }^{8}$ T. Shiomi, F. Hamada, and A. Nakajima, unpublished data. 
that observed except for the PDMS-cyclohexane system.

\section{DISCUSSION}

The purpose of this paper is to modify Flory's equation of state so as to predict the excess thermodynamic quantities by use of the temperatureindependent reduction parameters characteristic of each component. We tried to improve the discrepancy between experimental and calculated results for PDMS in various solvents.

For mixtures of $n$-alkanes, the FHN equation combined with temperature-independent reduction parameters gives results as good as the Flory equation with the reduction parameters dependent on temperature. Thus, the FHN equation satisfies fairly well the corresponding states principle.

For solutions of PDMS in various solvents, the six equations of state give almost the same results, i.e., the calculated results largely deviate from the experimental ones. This is contrast to the fact that the calculated results for pure liquids vary depending on the equation of state, as shown in Figures 14 and 8 in the preceding paper. Therefore, the discrepancy in PDMS solutions will be due to a cause other than the equation of state. The calculations for mixtures were carried out assuming the validity of the combining rules for mixing given by Flory. These rules seem too simple especially for polymer solutions. Therefore, further investigation in this point will be presented in another paper. ${ }^{14-16}$

\section{REFERENCES}

1. K. Fujisawa, T. Shiomi, F. Hamada, and A. Nakajima, Polym. J., 13, 993 (1981).

2. P. J. Flory, J. Am. Chem. Soc., 87, 1833 (1965).

3. B. E. Eichinger and P. J. Flory, Trans. Faraday Soc., 64, 2035 (1968)

4. P. J. Flory, Disc. Faraday Soc., 49, 7 (1970).

5. E. A. Guggenheim, Mol. Phys., 9, 199 (1965).

6. H. Reiss, H. L. Frisch, and J. L. Lebowitz, J. Chem. Phys., 31, 369 (1959); E. H. Helfand and H. L. Frisch, J. Chem. Phys., 34, 1037 (1961).

7. E. Thiele, J. Chem. Phys., 39, 474 (1963).

8. P. J. Flory and H. Shih, Macromolecules, 5, 761 (1972)

9. R. Chahal, W. Kao, and D. Patterson, J. Chem. Soc. Faraday Trans. 1, 69, 1834 (1973).

10. K. Sugamiya, N. Kuwahara, and M. Kaneko, Macromolecules, 7, 66 (1974).

11. T. Shiomi, Z. Izumi, F. Hamada, and A. Nakajima, Macromolecules, 13, 1149 (1980).

12. T. Shiomi, Y. Kohra, F. Hamada, and A. Nakajima, Macromolecules, 13, 1154 (1980).

13. R. A. Orwoll and P. J. Flory, J. Am. Chem. Soc., 89, 6822 (1967).

14. K. Fujisawa, F. Hamada, and A. Nakajima, Polym. Bull., 3, 261 (1980).

15. T. Shiomi, K. Fujisawa, F. Hamada, and A. Nakajima, J. Chem. Soc., Faraday Trans. 2, 76, 895 (1980)

16. F. Hamada, T. Shiomi, K. Fujisawa, and A. Nakajima, Macromolecules, 13, 729 (1980). 